\title{
ON NUMERICAL SIMULATION OF LIQUID POLYMER MOULDING ${ }^{1}$
}

\author{
R. ČIEGIS ${ }^{1}$ and O. ILIEV ${ }^{2}$ \\ ${ }^{1}$ Vilnius Gediminas Technical University \\ Saulètekio al. 11, LT-2040, Vilnius, Lithuania \\ E-mail: rc@fm.vtu. It \\ ${ }^{2}$ Fraunhofer ITWM \\ Europaallee 10, D-67657 Kaiserslautern, Germany \\ E-mail: iliev@itwm.fhg.de
}

Received June 20, 2003; revised October 3, 2003

\begin{abstract}
In this paper we consider numerical algorithms for solving the system of nonlinear PDEs, arising in modeling of liquid polymer injection. We investigate the particular case when a porous preform is located within the mould, so that the liquid polymer is flowing through a porous medium during the filling stage. The nonlinearity of the governing system of PDEs is due to the non-Newtonian behavior of the polymer, as well as due to the moving free boundary. The last is related to the penetration front, and a Stefan type problem is formulated to account for it. A finite-volume method is used to approximate the given differential problem. Results from numerical experiments are presented.

We also solve an inverse problem and present algorithms for determination of the absolute preform permeability coefficient for the case when the velocity of the penetration front is known from the measurements.

In both considered cases (direct and inverse problems) we emphasize on the specifics related to the non-Newtonian behavior of the polymer. For completeness, we discuss also the Newtonian case. Results of some experimental measurements are presented and discussed.
\end{abstract}

Key words: mathematical modeling, polymer moulding, finite-volume method

\footnotetext{
${ }^{1}$ This work was supported by DFG under the grant 269/13-1 and by the Lithuanian State Science and Studies Foundation within the framework of the Eureka project OPTPAPER EU-2623, E-2002.02.27
} 


\section{INTRODUCTION}

Composite materials are nowadays widely used in automotive, aerospace and many other industries. Liquid moulding technologies (see, e.g., [2]) are of strong economical interest for manufacturing high quality composite parts. The essence of the discussed technologies is that a dry fibrous mat, which form a porous preform, is located within the mould, and after that the liquid polymer is injected. In this way an accurate orientation of the fibers within the composite parts is achieved, making possible moulding parts with desired mechanical properties. In order to reduce the production costs of manufactured parts, mathematical modeling and numerical simulation are more and more extensively used at the designing stage. Because the filling of the mould is the most critical part of the process, most of th efforts are concentrated on its study. The review articles $[4 ; 17]$ give an impression about most of the mathematical models and numerical algorithms, developed in this area. The great part of the models (see, e.g., [4] and references therein) use the linear Darcy model to describe the liquid polymer flow through the porous preform, the last being considered in rigid body approximation. It should be noted, that such models are not valid for the flow of non-Newtonian fluids, also they do not account for the compressibility of the fibrous mat, and therefore these models have a limited area of applicability in modeling the polymer moulding. More advanced models consider either the coupled problem for Newtonian fluid in deformable porous media (see, e.g., $[1 ; 6 ; 7 ; 8$; $9 ; 17]$ ), or the flow of a non-Newtonian fluid in a rigid porous medium (see, e.g., [18]). The most complete formulation, concerning non-Newtonian flow in deformable porous medium is still not well studied.

Several challenging mathematical problems have to be solved in connection with simulation of liquid polymer moulding. Among them are developing of accurate numerical algorithms for solving the nonlinear free boundary direct problems, analysing and solving inverse problems (e.g., parameter estimation, etc.). This paper concerns several aspects of the modeling of LPM processes. These are:

(i) presenting a complete model for flow of non-Newtonian in deformable porous media;

(ii) solving an inverse problem for determining permeability in the case when the penetration front is known from the measurements;

(iii) developing a finite volume numerical algorithm for solving the 1-D direct problem.

First of all, a complete model for flow of non-Newtonian fluid in deformable porous media is listed. Curing (i.e., polymerization) is also accounted for. The model assumes sharp interface between the filled (wet) part of the porous preform and the unfilled (dry) part. A justification of this assumption for certain process regimes can be find, e.g., in [17]. For the approach dealing with an unsaturated subregion we refer to the discussion in [4]. In the next section we recall the 3 -D model from $[6 ; 7 ; 8 ; 9 ; 17]$ which treats Newtonian 
fluids, but accounts for the deformation of the preform and for the curing. The third section is devoted to a more detailed discussion of the model for the 1-D case. The fourth section concerns an extension of this model to the case of the non-Newtonian fluids.

Parameter (i.e., permeability) identification is discussed in Section 3 for the Newtonian case, and in Section 4 in the non-Newtonian case. Recall, that determination of the permeability is critical for the simulation of the filling. Once the permeability is known, the polymer injection can be simulated by analytical or numerical methods. Analytical solutions for the infiltration front position are presented in Section 5 for both cases: Newtonian and nonNewtonian fluids.

Section 6 is devoted to a numerical algorithm for solving the governing system of PDEs. Finite volume discretization, treating the moving boundaries, decoupling of the system, and results from some numerical experiments, are consecutively discussed there.

\section{MATHEMATICAL MODEL}

In this section we introduce the basic equations describing the injection moulding processes. Consider a deformable porous medium that at time $t=0$ starts being infiltrated. Let us denote by $D^{w}$ and $D^{d}$ the time-varying domains corresponding to the wet part of the solid preform (i.e., which is already wet by the infiltrating resin), and to the dry one (i.e., which is not yet reached by the liquid polymer), respectively.

We assume that capillary phenomena can be neglected, thus $D^{w}$ and $D^{d}$ are divided by a sharp interface $\sigma^{i}$ that represents the infiltration front. We also neglect the gravity force. The both assumptions are reasonable when the applied external pressure is relatively high. Let us denote by $\sigma^{e}$ the contact surface between the pure liquid and the wet solid.

The mathematical model consists of evolution equations for the state variables in the wet and in the dry regions, completed by interfaces conditions on $\sigma^{i}$ and $\sigma^{e}$ and by proper boundary conditions. Note, the general multicomponent mixture equations can be simplified [7] for the considered here case.

\subsection{Mathematical Model in the Wet Region}

The following variables are used to describe processes in the wet region:

$\Phi_{s}^{w}$ and $\Phi_{l}^{w}$ stand for the volume fraction occupied by the solid and the liquid constituents, respectively. Assuming the full saturation, the volume fraction occupied by the liquid satisfies the equality:

$$
\Phi_{l}^{w}=1-\Phi_{s}^{w} ;
$$

$\mathbf{v}_{s}^{w}, \mathbf{v}_{l}^{w}$ denote the velocities of the solid and the liquid constituents; 
$P_{l}^{w}$ is the pore liquid pressure.

$\theta^{w}$ is the temperature of the mixture in the wet region. Here we assume that the solid and liquid constituents there are locally in thermal equilibrium.

$\delta$ is the degree of cure of the resin. While penetrating the resin undergoes a polymerization process (i.e., curing), which is largely exothermic. It is defined as the ratio between the amount of heat released by the exothermic polymerization and the total heat of reaction. So $0 \leq \delta \leq 1$ (no curing for $\delta=0$, complete curing for $\delta=1$ ).

\subsubsection{Mass Conservation of Solid and Liquid Constituents}

Assuming that the solid and liquid are incompressible (i.e., the densities of the solid $\rho_{s}$ and liquid $\rho_{l}$ are constant), we obtain the local mass conservation equations in the Eulerian framework:

$$
\begin{aligned}
& \frac{\partial \Phi_{s}^{w}}{\partial t}+\nabla \cdot\left(\Phi_{s}^{w} \mathbf{v}_{s}^{w}\right)=0 \\
& \frac{\partial \Phi_{l}^{w}}{\partial t}+\nabla \cdot\left(\Phi_{l}^{w} \mathbf{v}_{l}^{w}\right)=0 .
\end{aligned}
$$

Assuming saturation, the volume fraction occupied by the liquid is given by

$$
\Phi_{l}^{w}=1-\Phi_{s}^{w}
$$

Summing up Eqs. (2.1) and (2.2) and introducing the composite velocity (or volume average velocity)

$$
\mathbf{v}_{c}^{w}=\Phi_{s}^{w} \mathbf{v}_{s}^{w}+\Phi_{l}^{w} \mathbf{v}_{l}^{w}
$$

and recalling the saturation condition (2.3) results in the equation

$$
\nabla \cdot \mathbf{v}_{c}^{w}=0
$$

Let us denote by $\rho_{m}^{w}$ the density of the mixture as a whole, i.e.:

$$
\rho_{m}^{w}=\rho_{s} \Phi_{s}^{w}+\rho_{l} \Phi_{l}^{w}
$$

and by $\mathbf{v}_{m}^{w}$ the velocity of the mixture

$$
\mathbf{v}_{m}^{w}=\frac{\rho_{s} \Phi_{s}^{w} \mathbf{v}_{s}^{w}+\rho_{l} \Phi_{l}^{w} \mathbf{v}_{l}^{w}}{\rho_{m}^{w}}
$$

Summing up Eqs. (2.1) and (2.2) multiplied by the corresponding densities gives the following mass conservation equation

$$
\frac{\partial \rho_{m}^{w}}{\partial t}+\nabla \cdot\left(\rho_{m}^{w} \mathbf{v}_{m}^{w}\right)=0
$$




\subsubsection{Momentum Balance Equations}

We will not consider general momentum balance equations. To focus on flow in porous media, the following simplifying assumptions are used [7]:

(A1) Negligible surface tension and capillary effects and slow liquid flow in the porous medium;

(A2) Negligible liquid excess-stress; excess interaction force between the solid and the liquid is proportional to the velocity difference $\mathbf{v}_{l}^{w}-\mathbf{v}_{s}^{w}$;

(A3) Negligible inertia if compared with the stresses; external body forces (e.g. gravity) are neglected;

Then we write the general momentum balance equations as

$$
\begin{aligned}
& \mathbf{v}_{l}^{w}-\mathbf{v}_{s}^{w}=-\frac{\mathbf{K}}{\mu \Phi_{l}^{w}} \nabla P_{l}^{w} \\
& \nabla P_{l}^{w}-\nabla \cdot \mathbf{T}_{m}=0
\end{aligned}
$$

where $\mu$ is the liquid viscosity, which depends on the degree of cure $\delta$ and on the temperature $\theta^{w}$

$$
\mu=\mu\left(\theta^{w}, \delta\right),
$$

and $\mathbf{K}$ is the permeability tensor, which for saturated deformable porous media depends on the deformation gradient $\mathbf{F}_{s}$ of the solid constituent $\mathbf{K}=\mathbf{K}\left(\mathbf{F}_{s}\right)$. $P_{l}^{w}$ is the pore liquid pressure, and $\mathbf{T}_{m}$ is the effective stress tensor.

Equation (2.5) is known as Darcy's law for deformable porous medium. A critical discussion of the hypotheses underlying Darcy's law is given in [14; 15]. Several generalizations of Darcy's law can be used here, e.g. Forchheimer law to account for fast flows, or correction to take into account the non-Newtonian properties of the resin (the last is discussed in the next sections).

To complete the model, one has to specify the constitutive equation for the stress tensor $\mathbf{T}_{m}$.

\subsubsection{Energy Balance}

Following the same procedure, which was used in previous above, it is possible to write the energy equation for the mixture:

$$
\begin{array}{r}
\rho_{m} c_{m}\left(\frac{\partial \theta^{w}}{\partial t}+\mathbf{v}_{m}^{w} \cdot \nabla \theta^{w}\right)=\nabla \cdot\left(\Lambda_{m}^{w} \nabla \theta^{w}\right)+\frac{1}{\mu} \mathbf{K} \nabla P^{w} \cdot \nabla P^{w} \\
+\Phi_{l}^{w} H_{c} f_{c}\left(\delta, \theta^{w}\right)-\frac{\rho_{s} \rho_{l} \Phi_{s}^{w} \Phi_{l}^{w}}{\rho_{m}}\left(c_{l}-c_{s}\right)\left(\mathbf{v}_{l}^{w}-\mathbf{v}_{s}^{w}\right) \nabla \theta^{w}
\end{array}
$$

where $c_{m}$ is the specific heat of the mixture:

$$
c_{m}=\frac{\rho_{s} \Phi_{s}^{w} c_{s}+\rho_{l} \Phi_{l}^{w} c_{l}}{\rho_{m}}
$$


$\Lambda_{m}^{w}$ is the thermal conductivity tensor of the mixture as the whole, the term $\Phi_{l}^{w} H_{c} f_{c}\left(\delta, \theta^{w}\right)$ represents the heat supplied by the curing reaction of the resin and the last term represents the heat diffusion due to the relative motion.

\subsubsection{The Degree of Curing}

As the liquid is moving, the evolution of the degree of cure is modeled by the equation

$$
\frac{\partial \delta}{\partial t}+\mathbf{v}_{l}^{w} \cdot \nabla \delta=f_{c}\left(\delta, \theta^{w}\right),
$$

where $f_{c}$ is an experimentally determined function describing the reaction.

\subsection{Mathematical Model in the Dry Region}

We proceed in a way similar to the one outlined for the wet region. However some additional assumptions can be used, which enable us to simplify the model.

(D1) The air pressure is everywhere equal to the atmospheric pressure;

(D2) The gas contribution to the global stress may be neglected;

(D3) The average velocity is equal to the velocity of the solid constituent and the composite density $\rho_{m} \approx \Phi_{s}^{d} \rho_{s}$.

Thus we have the following state variables in the dry region: $\Phi_{s}^{d}$ is the solid volume fraction; $\mathbf{v}_{s}^{d}$ is the solid velocity; $\theta^{d}$ is the temperature.

Mass Conservation

$$
\frac{\partial \Phi_{s}^{d}}{\partial t}+\nabla \cdot\left(\Phi_{s}^{d} \mathbf{v}_{s}^{d}\right)=0 .
$$

Momentum Balance Equation

$$
\nabla \cdot \mathbf{T}_{s}=0
$$

where $\mathbf{T}_{s}$ is the stress tensor of the dry solid. Here we assumed, that $\mathbf{T}_{m}=\mathbf{T}_{s}$.

To complete the model we still have to specify the constitutive equations for the stress tensors $\mathbf{T}_{m}$ and $\mathbf{T}_{s}$. We will assume that the wet and dry solids behave elastically.

\section{Energy Balance Equation}

$$
\rho_{s} \Phi_{s}^{d} c_{s}\left(\frac{\partial \theta^{d}}{\partial t}+\mathbf{v}_{s}^{d} \cdot \nabla \theta^{d}\right)=\nabla \cdot\left(\Lambda_{s}^{w} \nabla \theta^{d}\right)
$$

where $\boldsymbol{\Lambda}_{s}^{w}$ is the thermal conductivity of the solid. 


\subsection{Interface and Boundary Conditions}

Infiltration Front

Let the infiltration interface $\sigma^{i}$ be given by the surface

$$
\psi_{i}(x, y, z, t)=0 \text {. }
$$

This surface moves together with the propagation of the liquid, thus its evolution equation is given by

$$
\frac{\partial \psi_{i}}{\partial t}+\mathbf{v}_{l}^{w}\left(\sigma^{i}\right) \cdot \nabla \psi_{i}=0
$$

Preform Border

Let the contact surface $\sigma^{e}$ between the liquid and the wet solid be given by

$$
\psi_{e}(x, y, z, t)=0 \text {. }
$$

As the resin penetrates the porous solid this material surface is fixed on the solid, and therefore its evolution equation is

$$
\frac{\partial \psi_{e}}{\partial t}+\mathbf{v}_{s}^{w}\left(\sigma^{e}\right) \cdot \nabla \psi_{e}=0
$$

Jump Conditions for Material Surfaces

Considering the mixture as a whole, the following jump conditions are obtained for material surfaces $[7 ; 8 ; 13]$

$$
\begin{aligned}
& {\left[\rho_{m}\left(\mathbf{v}_{m}-\mathbf{v}_{\sigma}\right)\right] \cdot \mathbf{n}_{\sigma}=0,} \\
& {[\theta]=0,} \\
& {\left[-P \mathbf{I}+\mathbf{T}_{m}\right] \cdot \mathbf{n}_{\sigma}=0,} \\
& {[P]=0}
\end{aligned}
$$

where $\mathbf{n}_{\sigma}$ is the normal outside $D^{w}$. It follows from (2.14) that

$$
\left[\mathbf{v}_{c}\right] \cdot \mathbf{n}_{\sigma}=0 .
$$

Using (2.17), (2.16) gives the continuity of the stress $\mathbf{T}_{m}$ across the the surface:

$$
\left[\mathbf{T}_{m}\right] \cdot \mathbf{n}_{\sigma}=0
$$

In one-dimensional case assuming the same constitutive equation of elastic type for wet and dry solids, this implies the continuity of $\Phi_{s}$ across $\sigma^{i}$, and then we get from (2.17) the continuity of $\mathbf{v}_{s}$.

If the specific heat of the solid is continuous across $\sigma^{i}$, the temperature fluxes satisfy the following condition:

$$
\left[\boldsymbol{\Lambda}_{m} \nabla \theta\right] \cdot \mathbf{n}_{\sigma}=0
$$


Boundary Conditions on $\sigma^{e}$

Let the superscript ${ }^{-}$denotes the quantities evaluated in the pure liquid region. Then we have the following conditions

$$
\begin{aligned}
& \Phi_{s}^{-}=0, \quad \mathbf{v}_{l}^{-}=\mathbf{v}_{i n}, \\
& \mathbf{T}^{-} \mathbf{n}_{\sigma^{e}}=0, \quad P^{-}=P_{0},
\end{aligned}
$$

where $\mathbf{v}_{i n}$ is the inflow velocity of the resin and $P_{0}$ the pressure driving the flow. Thus in the wet region we have $\Phi_{s}^{w}\left(\sigma^{e}, t\right)=\Phi_{r}$, where $\Phi_{r}$ is the solid volume fraction in the dry undeformed preform.

The temperature on $\sigma^{e}$ is $\theta=\theta_{i n}$, where $\theta_{i n}$ is the temperature of the infiltrating liquid.

Boundary Conditions for the Curing Equation

The curing equation (2.8) is hyperbolic thus the boundary conditions

$$
\delta\left(\sigma^{e}\right)=\delta_{i n}
$$

must be specified on the part of the boundary where the characteristics enter the domain (the resin enters the preform), i.e. where $\left(\mathbf{v}_{l}^{w}-\mathbf{v}_{s}^{w}\right) \cdot \mathbf{n}_{\sigma^{e}}<0$.

\section{ONE-DIMENSIONAL INFILTRATION}

This section deals with one-dimensional problems (see $[1 ; 6 ; 7 ; 8]$ ). Assume that the porous medium is initially dry, homogeneous, isotropic and that the flow and the strain take place only along $x$ axis.

Let us denote by $x=x_{e}(t)$ the left border of the preform, which can move (due to the preform's compression) when the liquid touches it. The infiltration front $x=x_{i}(t)$ separates the wet region $D^{w}$ from the remaining dry region $D^{d}$ :

$$
D^{w}=\left[x_{e}(t), x_{i}(t)\right], \quad D^{d}=\left[x_{i}(t), L\right] .
$$

As infiltration proceeds, both the dry and the wet preforms compress or expand back according to the process conditions.

The one-dimensional mathematical model is obtained from the system of equations given in the previous section.

For $t \leq 0$ the whole preform is dry, at rest, and compressed at a given volume ratio:

$$
\left\{\begin{array}{l}
\Phi_{s}^{d}(x, t=0)=\Phi_{r}, \quad x \in[0, L], \\
x_{e}(t=0)=0, x_{i}(t=0)=0,
\end{array}\right.
$$

where $\Phi_{r}$ is the solid volume fraction of the solid preform in its undeformed configuration. 


\subsection{Wet Region}

In the wet region the following equations are satisfied:

$$
\begin{aligned}
& \frac{\partial \Phi_{s}^{w}}{\partial t}+\frac{\partial}{\partial x}\left(\Phi_{s}^{w} v_{s}^{w}\right)=0 \\
& \Phi_{s}^{w}\left(x_{e}(t), t\right)=\Phi_{r}, \quad t>0 \\
& \frac{\partial v_{c}^{w}}{\partial x}=0 \\
& \left(1-\Phi_{s}^{w}\right)\left(v_{l}^{w}-v_{s}^{w}\right)=-\frac{K}{\mu\left(\delta, \theta^{w}\right)} \frac{\partial P^{w}}{\partial x}, \\
& \frac{\partial P^{w}}{\partial x}+\frac{\partial \tau^{w}}{\partial x}=0, \\
& \rho_{m} c_{m}\left(\frac{\partial \theta^{w}}{\partial t}+v_{s}^{w} \frac{\partial \theta^{w}}{\partial x}\right)=\frac{\partial}{\partial x}\left(\lambda_{m} \frac{\partial \theta^{w}}{\partial x}\right)-\rho_{l} c_{l} \frac{K}{\mu\left(\delta, \theta^{w}\right)} \frac{\partial \tau^{w}}{\partial x} \frac{\partial \theta^{w}}{\partial x} \\
& \quad+\left(1-\Phi_{s}^{w}\right) H_{c} f_{c}\left(\delta, \theta^{w}\right)+\frac{K}{\mu\left(\delta, \theta^{w}\right)}\left(\frac{\partial \tau^{w}}{\partial x}\right)^{2}-\tau^{w} \frac{\partial v_{s}^{w}}{\partial x} \\
& \frac{\partial \delta}{\partial t}+v_{l}^{w} \frac{\partial \delta}{\partial x}=f_{c}\left(\delta, \theta^{w}\right)
\end{aligned}
$$

where $\tau^{w}$ is the $x x$ component of $\mathbf{T}_{m}$ :

$$
\tau^{w}=-\left(\mathbf{T}_{m}\right)_{x x}, x \in D^{w}, t \geq 0,
$$

and $K$ is the $x x$ component of the permeability tensor $K=(\mathbf{K})_{x x}$.

Velocity Driven Infiltration

Before formulating equations in the dry region $D^{d}$ we will use equation (3.2), from which it follows that $v_{c}$ is space independent in $D^{w}$

$$
v_{c}(x, t)=v(t) \text {. }
$$

Due to jump conditions, formulated in previous section, the composite velocity is continuous across $x_{e}(t)$, thus we have:

$$
v_{c}(x, t)=u_{i n}(t), \quad x \in D^{w},
$$

where $u_{i n}(t)$ is the velocity of the infiltration liquid.

Using Darcy's law (3.3) we can express the velocities of the solid and liquid constituents

$$
\begin{aligned}
& v_{s}^{w}=u_{i n}+\frac{K}{\mu} \frac{\partial P^{w}}{\partial x} \\
& v_{l}^{w}=u_{i n}-\frac{\Phi_{s}^{w}}{1-\Phi_{s}^{w}} \frac{K}{\mu} \frac{\partial P^{w}}{\partial x}
\end{aligned}
$$


The infiltration front $x_{i}(t)$ moves with the liquid, thus we have the initial value problem

$$
\left\{\begin{array}{l}
\frac{d x_{i}(t)}{d t}=v_{l}^{w}\left(x_{i}(t), t\right)=u_{i n}-\frac{\Phi_{s}^{w}}{1-\Phi_{s}^{w}} \frac{K}{\mu} \frac{\partial P^{w}}{\partial x} \\
x_{i}(t=0)=0
\end{array}\right.
$$

Determination of the Permeability

It follows from (3.7) that the permeability of the homogeneous structure can be determined as

$$
K=\left(u_{i n}-v_{l}^{w}\right) \frac{\left(1-\Phi_{s}^{w}\right) \mu}{\Phi_{s}^{w}}\left(\frac{\partial P^{w}}{\partial x}\right)^{-1}
$$

if experimental measurements of the interface velocity $v_{l}^{w}\left(x_{i}(t), t\right)$, the volume fraction occupied by the solid $\Phi_{s}^{w}$, and pressure gradients are available.

\section{Constitutive Models}

We still need to specify the constitutive equations for the stresses. Two models can be used.

(WA1) Considering the elastic model for wet porous medium preform means that

$$
\tau^{w}=\Sigma_{w}\left(\Phi_{s}^{w}\right),
$$

where $\Sigma_{w}$ is a strictly increasing function. Most frequently it is assumed that

$$
\Sigma_{d}=\Sigma_{w} .
$$

Then the continuity of the stress across $x_{i}$ implies also the continuity of the $\Phi_{s}$. If wet and dry preforms behave elastically with $\Sigma_{d} \neq \Sigma_{w}$, then the continuity of $\Phi_{s}$ across $x_{i}$ does not hold any more.

(WA2) Due to the fact that the solid and the liquid matrices can not deform independently but have to carry the load by joint deformations, the wet preform is modeled by the linear model

$$
\begin{aligned}
\lambda\left(\frac{\partial \tau^{w}}{\partial t}\right. & \left.+v_{s}^{w} \frac{\partial \tau^{w}}{\partial x}-2 a \tau^{w} \frac{\partial v_{s}^{w}}{\partial x}\right)+\tau^{w}=\Lambda\left(\frac{\partial \Sigma_{w}\left(\Phi_{s}^{w}\right)}{\partial t}\right. \\
& \left.+v_{s}^{w} \frac{\partial \Sigma_{w}\left(\Phi_{s}^{w}\right)}{\partial x}-2 a \frac{\partial v_{s}^{w}}{\partial x} \Sigma_{w}\left(\Phi_{s}^{w}\right)\right)+\Sigma_{w}\left(\Phi_{s}^{w}\right)
\end{aligned}
$$

where $\lambda$ is called the relaxation time, $\Lambda$ is the retardation time, $\Lambda \geq \lambda$ and $a$ is a parameter ranging in $[-1,1]$. 
(DA3) The dry preform is always assumed to behave elastically

$$
\tau^{d}=\Sigma_{d}\left(\Phi_{s}^{d}\right)
$$

where $\Sigma_{d}$ is a strictly increasing function of the solid volume fraction.

\subsection{Dry Region}

Since the interaction between the air and the solid can be assumed negligible, there is no pressure drop in the air. Therefore $P^{d}(x, t)=P_{a t m}$, where $P_{a t m}$ is the atmospheric pressure.

Let $\tau^{d}$ be the $x x$ component of the excess stress tensor in the dry region, then we obtain

$$
\frac{\partial \tau^{d}}{\partial x}=0
$$

If the dry preform is assumed to behave elastically, then equations (3.8) and (3.9) imply that $\Phi_{s}^{d}$ is space independent.

$$
\Phi_{s}^{d}=\Phi_{s}^{d}(t)
$$

Due to continuity of $v_{c}$ across the interface $x_{i}(t)$ we have

$$
v_{c}^{w}\left(x_{i}(t), t\right)=v_{c}^{d}\left(x_{i}(t), t\right)
$$

or

$$
v_{c}(x, t)=u_{i n}(t), \quad x \in D^{d} .
$$

Then from the continuity equation and from the boundary condition

$$
v_{s}^{d}(L, t)=0
$$

(i.e. the preform is constrained by a fixed draining boundary at $x=L$ ) and using the fact that $\Phi_{s}^{d}$ is space independent one has

$$
v_{s}^{d}(x, t)=\frac{\frac{d}{d t}\left(\Phi_{s}^{d}(t)\right)}{\Phi_{s}^{d}}(L-x)
$$

Neglecting the influence of the air, we get the simplified heat equation

$$
\rho_{s} c_{s}\left(\Phi_{s}^{d} \frac{\partial \theta^{d}}{\partial t}+\frac{d \Phi_{s}^{d}}{d t}(L-x) \frac{\partial \theta^{d}}{\partial x}\right)=\frac{\partial}{\partial x}\left(\Phi_{s}^{d} \lambda_{s} \frac{\partial \theta^{d}}{\partial x}\right)
$$


Pressure Driven Infiltration

If the inlet pressure $\Delta P(t)=P_{0}(t)-P_{a t m}$ is given, then we can integrate the momentum equation (3.4) and use the continuity of the stress on the infiltration front:

$$
\tau^{d}(t)=\Delta P(t), \quad t>0 .
$$

Then the solid volume fraction of the dry region is given as

$$
\Phi_{s}^{d}(t)=\Sigma_{d}^{-1}(\Delta P(t))
$$

Using this formula and the mass conservation we can find the initial position of the left border of the preform after incoming liquid compresses the preform:

$$
x_{e}(0)=L\left(1-\frac{\Phi_{r}}{\Phi_{s}^{d}(0)}\right)
$$

Let denote by $Q$ the function

$$
Q=-\frac{K\left(\Phi_{s}^{w}\right)}{\mu} \frac{\partial P}{\partial x}
$$

Evaluating the composite velocity $v_{c}$ on both sides of the infiltration front $x_{i}(t)$ and recalling the fact that $x_{i}(t)$ is a material interface fixed on the liquid phase, one can write the equation

$$
\begin{aligned}
u_{i n} & =\Phi_{s}^{w}\left(u_{i n}-Q\right)+\left(1-\Phi_{s}^{w}\right) \frac{d x_{i}}{d t} \\
& =-\frac{d}{d t}\left(\left(1-\Phi_{s}^{d}\right)\left(L-x_{i}\right)\right)
\end{aligned}
$$

Using (3.7) we can eliminate $u_{\text {in }}$ from the obtained equation, this gives the initial value problem for the interface $x_{i}(t)$ :

$$
\left\{\begin{array}{l}
\frac{d\left(\Phi_{s}^{d} x_{i}\right)}{d t}=L \frac{d \Phi_{s}^{d}}{d t}+\left(\frac{\Phi_{s}^{w} Q}{1-\Phi_{s}^{w}}\right)\left(x_{i}(t), t\right), \\
x_{i}(0)=x_{i 0}
\end{array}\right.
$$

The inflow velocity is then determined as

$$
u_{i n}(t)=\left(L-x_{i}(t)\right) \frac{\left(\Phi_{s}^{d}\right)^{\prime}}{\Phi_{s}^{d}}+\left(\frac{1}{\Phi_{s}^{d}}-1\right)\left(\frac{\Phi_{s}^{w} Q}{1-\Phi_{s}^{w}}\right)\left(x_{i}(t), t\right) .
$$

If $\Delta P(t)$ is constant in time, then after simple computations it can be proved that

$$
u_{i n}(t)=\left(1-\Phi_{s}^{d}\right) \frac{d x_{i}(t)}{d t}
$$




\section{NON-NEWTONIAN FLOW}

In previous section we have considered the Newtonian fluids, for which Darcy's law specifies the relation between the velocity and the pressure. But a large number of fluids, such as polymer solutions, polymer melts, suspensions do not follow Newton's law of viscosity [10]. One of the possibilities to describe non-Newtonian fluids is to modify the classical Darcy's law and to use a generalized Darcy's law (power law). For one-dimensional case we have:

$$
\left(1-\Phi_{s}^{w}\right)\left(v_{l}^{w}-v_{s}^{w}\right)=\left(-\frac{K}{H} \frac{\partial P^{w}}{\partial x}\right)^{1 / n}
$$

where $n$ is used to describe different types of fluids. If $n<1$ then a fluid is pseudoplastic (polymer solutions are pseudoplastic). A dilatant fluids are described using $n>1$. Here $H$ is the non-Newtonian bed factor. Following [3] we combine a power law model of a non-Newtonian fluid with the BlakeKozeny model for the porous medium, this gives

$$
H=\frac{\mu}{12}\left(9+\frac{3}{n}\right)^{n}(150 K \epsilon)^{(1-n) / 2},
$$

where $\epsilon$ is the porosity of the structure. Then equation (4.1) can be rewritten as:

$$
\begin{aligned}
& \left(1-\Phi_{s}^{w}\right)\left(v_{l}^{w}-v_{s}^{w}\right)=\left(-\frac{K^{(1+n) / 2}}{\mu d_{n}} \frac{\partial P^{w}}{\partial x}\right)^{1 / n} \\
& d_{n}=\frac{1}{12}\left(9+\frac{3}{n}\right)^{n}(150 \epsilon)^{(1-n) / 2}
\end{aligned}
$$

Determination of the Permeability

Using the generalized Darcy's law (4.2) and the fact that $v_{c}$ is constant in space we can express the velocity of the liquid constituent

$$
v_{l}^{w}=u_{i n}+\frac{\Phi_{s}^{w}}{1-\Phi_{s}^{w}}\left(-\frac{K^{(1+n) / 2}}{\mu d_{n}} \frac{\partial P^{w}}{\partial x}\right)^{1 / n} .
$$

The infiltration front $x_{i}(t)$ moves with the liquid, thus we have the initial value problem

$$
\left\{\begin{array}{l}
\frac{d x_{i}(t)}{d t}=v_{l}^{w}\left(x_{i}(t), t\right)=u_{i n}+\frac{\Phi_{s}^{w}}{1-\Phi_{s}^{w}}\left(-\frac{K^{(1+n) / 2}}{\mu d_{n}} \frac{\partial P^{w}}{\partial x}\right)^{1 / n} \\
x_{i}(t=0)=0
\end{array}\right.
$$


After measuring the front velocity $v_{l}^{w}\left(x_{i}(t), t\right)$, the volume fraction corresponding to the solid $\Phi_{s}^{w}$ and the pressure gradiant, we can determine the permeability of the homogeneous structure as

$$
K=\left(-\left(\left(v_{l}^{w}-u_{i n}\right) \frac{1-\Phi_{s}^{w}}{\Phi_{s}^{w}}\right)^{n} \mu d_{n}\left(\frac{\partial P^{w}}{\partial x}\right)^{-1}\right)^{2 /(1+n)}
$$

\section{ONE - PHASE MODEL}

It is well known that the macroscopic flow behavior at large length is well captured by one-phase flow models (see publications on RTM $[4 ; 16 ; 19]$ ). Such models are sufficiently accurate in predicting flow-front location, moldfilling time, and pressure distribution during mold filling.

In this section we consider one-dimensional model of the injection in a nondeformable porous media (i.e., a rigid preform) with a resin as the only phase. Its flow is governed by the Darcy's law

$$
q=-\frac{K}{\mu} \frac{\partial P(x, t)}{\partial x}
$$

or the generalized Darcy's law

$$
q=\left(-\frac{K^{(1+n) / 2}}{\mu d_{n}} \frac{\partial P(x, t)}{\partial x}\right)^{1 / n}
$$

where $q$ is the volumetric velocity (i.e., the amount of volume traversing a unit area per unit time through the preform). We also assume that injection is isothermal

If the flow is saturated, then the assumption of resin incompressibility (i.e., the mass balance) leads to the following boundary value problem:

$$
\left\{\begin{array}{l}
\frac{\partial q}{\partial x}=0 \\
P(0, t)=P_{0}(t), P\left(x_{i}(t), t\right)=P_{a t m}
\end{array}\right.
$$

We can obtain the solution in the explicit form (this formula is also valid for the generalized Darcy's law):

$$
P(x, t)=P_{0}(t)+\frac{P_{a t m}-P_{0}(t)}{x_{i}(t)} x .
$$

Let consider the Darcy's law. The evolution the infiltration front is described by the initial value problem 


$$
\left\{\begin{array}{l}
\frac{d x_{i}(t)}{d t}=\frac{K}{\mu} \frac{P_{0}(t)-P_{a t m}}{x_{i}(t)} \\
x_{i}(0)=0
\end{array}\right.
$$

If the driving pressure is constant in time, then we obtain the position of the infiltration front in the explicit form:

$$
x_{i}(t)=\sqrt{\frac{2 K}{\mu}\left(P_{0}-P_{a t m}\right) t} .
$$

Similarly for the generalized Darcy's law the infiltration front is given by

$$
x_{i}(t)=\left(\frac{K^{(1+n) / 2}}{\mu d_{n}}\left(P_{0}-P_{a t m}\right)\right)^{1 /(n+1)}\left(\frac{n+1}{n} t\right)^{n /(n+1)} .
$$

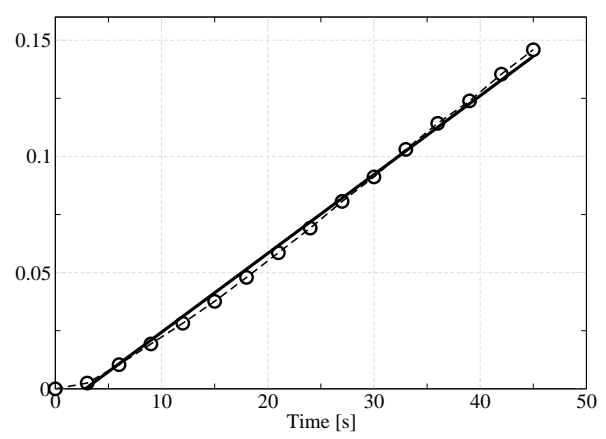

Figure 1. Position of $x_{i}^{2}(t)$ : measured values (circles) and a fitted curve (solid line).

Experimental Data. In this paragraph we present certain results of experimental measurements performed in the University of Kaiserslautern. The conditions of the experiment were characterized by a slow flow, a Newtonian liquid was used in experiments. In Figure 1 we plot the measured values of $x_{i}^{2}(t)$ (circles) and the linear approximation (solid line), which is fitted to the experimental data using the Least Squares method. As it can be expected, the presented results show that the model based on Darcy's law gives a good approximation of the movement of the infiltration front in this case. Measurements with a non-Newtonian liquid are to be performed and compared with our analytical predictions in the near future. 


\section{NUMERICAL METHOD}

Let us assume that at time moment $t=0$ the liquid touches the left border of the preform. We are not trying to describe the early instants of the infiltration process and simply state that the incoming liquid compresses the preform and wets some its part, therefore the initial positions of the border $x_{e}$, as well of the infiltration front $x_{i}$ are given a priori

$$
x_{e}(0)=x_{e 0}, \quad x_{i}(0)=x_{i 0} .
$$

Then we can identify a wet region $D^{w}$ and a dry region $D^{d}$.

There are two main difficulties in constructing discrete approximations of the given differential problem:

- Moving boundaries $x_{e}(t)$ and $x_{i}(t)$ (the Stefan type problem);

- The generalized Darcy's law for flow velocities.

In this section we will analyze some methods for solving the described above one-dimensional differential problems. In order to simplify the presentation we restrict to the mass and momentum balance Eqs. (3.1)-(3.4):

$$
\left\{\begin{array}{l}
\frac{\partial \Phi_{s}^{w}}{\partial t}+\frac{\partial}{\partial x}\left(\Phi_{s}^{w} v_{s}^{w}\right)=0, \\
\Phi_{s}^{w}\left(x_{e}(t), t\right)=\Phi_{r}, \Phi_{s}^{w}\left(x_{i}(t), t\right)=\Phi_{m}, t>0, \\
\Phi_{s}^{w}(x, 0)=\Phi_{0}(x), x_{e}(t)<x<x_{i}(t), \\
\frac{\partial v_{c}^{w}}{\partial x}=0 \\
\left(1-\Phi_{s}^{w}\right)\left(v_{l}^{w}-v_{s}^{w}\right)=Q, \\
\frac{\partial P^{w}}{\partial x}+\frac{\partial \tau^{w}}{\partial x}=0,
\end{array}\right.
$$

here $Q$ describes the Darcy's or generalized Darcy's law terms.

\subsection{Discretization}

The given one-dimensional problem is defined in the region with two free boundaries. Following [1] we define discrete meshes which are dynamically adapted to the moving boundaries:

$$
\begin{aligned}
& D_{h}^{w}=\left\{x_{j}=x_{j}(t): x_{j}=x_{e}(t),+h_{w} j, j=0,1, \ldots, M, x_{M}=x_{i}(t)\right\}, \\
& D_{h}^{d}=\left\{x_{j}=x_{j}(t): x_{j}=x_{i}(t)+h_{d} j, j=M, M+1, \ldots, N, x_{N}=L\right\},
\end{aligned}
$$

here $M=M(t)$ is selected to preserve the quasi-uniform spatial discretization of the whole region $D$

$$
M=\frac{\left(x_{i}(t)-x_{e}(t)\right) N}{L}, \quad M \geq 1 .
$$


Let $\tau^{n}$ denotes the discrete time step at $t=t^{n}$. In the following we will denote by $\Phi_{j}^{n}$ the finite difference approximation of $\Phi_{s}^{w}\left(x_{j}, t^{n}\right)$.

\section{Infiltration Front}

The front tracking method is applied to determine the time step $\tau_{n}$. Using the free boundary equation for $x_{i}(t)$, the time step $\tau_{n}$ is chosen so that the infiltration front jumps from one node to the next node per time step. Thus the discrete mesh parameter $M\left(t^{n}\right)=n$ and the time step $\tau_{n}$ is computed from the following equation

$$
\frac{L-x_{e}(0)}{N \tau_{n}}=\frac{\Phi_{M-\frac{1}{2}}^{n}}{\left(1-\Phi_{M-\frac{1}{2}}^{n}\right) \Phi_{s}^{d}} Q\left(x, t^{n}\right)_{M-\frac{1}{2}} .
$$

\section{Approximation of the Differential Equations}

For a moment let us assume that the left boundary $x_{e}(t)$ is not moving, i.e. remains constant. In the case of Darcy's law we can reduce the system of equations to a single parabolic problem [9]

$$
\left\{\begin{array}{l}
\frac{\partial \Phi_{s}^{w}}{\partial t}+u_{i n}(t) \frac{\partial \Phi_{s}^{w}}{\partial x}=\frac{\partial}{\partial x}\left(\frac{K\left(\Phi_{s}^{w}\right)}{\mu} \frac{d \Sigma_{w}\left(\Phi_{s}^{w}\right)}{d \Phi_{s}^{w}} \Phi_{s}^{w} \frac{\partial \Phi_{s}^{w}}{\partial x}\right) \\
\Phi_{s}^{w}\left(x_{e}, t\right)=\Phi_{r}, \Phi_{s}^{w}\left(x_{i}(t), t\right)=\Phi_{m}, \quad t>0 .
\end{array}\right.
$$

Let us introduce the following notation of finite differences:

$$
\delta_{-} \Phi_{j}=\frac{\Phi_{j}-\Phi_{j-1}}{h}, \quad \delta_{+} \Phi_{j}=\frac{\Phi_{j+1}-\Phi_{j}}{h} .
$$

The discrete approximation is obtained using the finite-volume method. We use the upwind approximation for the convection part, centered differencing for the diffusion part and a backward Euler method for integration in time, then the difference scheme takes the following form

$$
\left\{\begin{aligned}
\frac{\Phi_{j}^{n+1}-\tilde{\Phi}_{j}^{n}}{\tau_{n}}+ & u_{i n}^{n+1} \delta_{-} \Phi_{j}^{n+1} \\
& =\frac{a_{j+0.5}\left(\Phi^{n+1}\right) \delta_{+} \Phi_{j}^{n+1}-a_{j-0.5}\left(\Phi^{n+1}\right) \delta_{-} \Phi_{j}^{n+1}}{h} \\
\Phi_{0}^{n+1}=\Phi_{r}, & \Phi_{M}^{n+1}=\Phi_{m},
\end{aligned}\right.
$$

where

$$
\tilde{\Phi}_{j}^{n}= \begin{cases}\Phi_{j}^{n}, & 1 \leq j<M-1 \\ \frac{1}{2}\left(\Phi_{M-1}^{n}+\Phi_{M}^{n}\right), & j=M-1\end{cases}
$$


The system of nonlinear equations is solved using the Picard linearization:

$$
\left\{\begin{aligned}
\frac{\Phi_{j}^{n, r}-\tilde{\Phi}_{j}^{n}}{\tau_{n}}+ & u_{i n}^{n+1} \delta_{-} \Phi_{j}^{n, r} \\
& =\frac{a_{j+0.5}\left(\Phi^{n, r-1}\right) \delta_{+} \Phi_{j}^{n, r}-a_{j-0.5}\left(\Phi^{n, r-1}\right) \delta_{-} \Phi_{j}^{n, r}}{h} \\
\Phi_{0}^{n, r}=\Phi_{r}, & \Phi_{M}^{n, r}=\Phi_{m} \\
\Phi_{j}^{n, 0}=\Phi_{j}^{n}, & j=1,2, \ldots, M-1
\end{aligned}\right.
$$

Generalized Darcy's Law

The given above algorithm also can be used if the flow velocities are described by the generalized Darcy's law, at least when the parameter $n_{D}<1$. Here we have changed the notation of $n$ to $n_{D}$ in formula (5.2). Then the iterative algorithm takes the following form:

$$
\begin{aligned}
& \frac{\Phi_{j}^{n, r}-\Phi_{j}^{n}}{\tau_{n}}+u_{i n}^{n+1} \delta_{-} \Phi_{j}^{n, r}=\frac{b_{j+0.5}\left(\Phi^{n, r-1}\right) \delta_{+} \Phi_{j}^{n, r}-b_{j-0.5}\left(\Phi^{n, r-1}\right) \delta_{-} \Phi_{j}^{n, r}}{h} \\
& b_{j+0.5}(\Phi)=\left(\left(\frac{K^{\left(1+n_{D}\right) / 2}(\Phi)}{\mu d_{n}} \frac{d \Sigma_{w}(\Phi)}{d \Phi}\right)^{\frac{1}{n_{D}}} \Phi\right)_{j+\frac{1}{2}}\left|\delta_{+} \Phi_{j}\right|^{\left(1-n_{D}\right) / n_{D}} .
\end{aligned}
$$

\subsection{Moving Boundaries}

In this section we will take into account that the contact surface $x_{e}(t)$ is fixed on the solid. Thus if $v_{s}\left(x_{e}(t), t\right) \neq 0$, then the boundary is also moving. Let us consider the elementary domain, which is described by our mesh $\left[x_{j-1}(t), x_{j}(t)\right]$. We denote by $v=v(x, t)$ the velocity of grid points. Then it is convenient to use the integral formulation of the mass balance equation

$$
\frac{\partial}{\partial t} \int_{x_{j-1}(t)}^{x_{j}(t)} \Phi_{s}^{w} d x+\frac{\partial}{\partial x} \int_{x_{j-1}(t)}^{x_{j}(t)}\left(v_{s}^{w}-v\right) \Phi_{s}^{w} d x=0 .
$$

Applying the finite-volume method we approximate the integral equation (6.3) by the following conservative finite-difference scheme

$$
\left\{\begin{array}{c}
\frac{h^{n, r} \Phi_{j}^{n, r}-h^{n} \tilde{\Phi}_{j}^{n}}{\tau_{n}}+F\left(w_{j+\frac{1}{2}}^{n, r}, \Phi_{j+1}^{n, r}, \Phi_{j}^{n, r}\right)-F\left(w_{j-\frac{1}{2}}^{n, r}, \Phi_{j}^{n, r}, \Phi_{j-1}^{n, r}\right) \\
=a_{j+\frac{1}{2}}\left(\Phi^{n, r-1}\right) \delta_{+} \Phi_{j}^{n, r}-a_{j-\frac{1}{2}}\left(\Phi^{n, r-1}\right) \delta_{-} \Phi_{j}^{n, r}, \\
w_{j+\frac{1}{2}}^{n, r}=u_{i n}^{n+1}-v_{j+\frac{1}{2}}^{n, r}
\end{array}\right.
$$


where the numerical flux $F\left(w_{j+\frac{1}{2}}, \Phi_{j+1}, \Phi_{j}\right)$ is defined as follows (see, [5; $12]):$

$$
F\left(w_{j+\frac{1}{2}}, \Phi_{j+1}, \Phi_{j}\right)=\frac{1}{2} w_{j+\frac{1}{2}}\left(\Phi_{j+1}+\Phi_{j}\right)-\frac{1}{2}\left|w_{j+\frac{1}{2}}\right|\left(\Phi_{j+1}-\Phi_{j}\right) .
$$

The velocity of the movement of the grid point $x_{j}\left(t^{n}\right)$ is defined as

$$
v_{j}\left(t^{n, r}\right)=v_{s}^{w}\left(x_{e}\left(t^{n, r}\right), t^{n, r}\right)\left(1-\frac{j}{M}\right), \quad j=0,1, \ldots, M
$$

and the contact surface $x_{e}\left(t^{n}\right)$ moves with the velocity:

$$
v_{s}^{w}\left(x_{e}\left(t^{n, r}\right)\right)=\frac{\left(u_{i n}^{n+1}-a_{1 / 2}\left(\Phi^{n, r-1}\right) \delta_{+} \Phi_{0}^{n, r-1}\right) \Phi_{1 / 2}^{n, r-1}+\frac{h^{n, r} \Phi_{0}^{n, r-1}-h^{n} \Phi_{0}^{n}}{2 \tau_{n}}}{\Phi_{0}^{n, r-1}} .
$$

The contact front position is updated by the following formula:

$$
\begin{aligned}
& w^{n, r}=\theta w^{n, r-1}+(1-\theta) v_{s}^{w}\left(x_{e}\left(t^{n, r}\right), t^{n, r}\right), \quad 0<\theta<1 \\
& x_{e}\left(t^{n, r}\right)=x_{e}\left(t^{n}\right)+\tau_{n} w^{n, r}, \quad w^{n, 0}=0
\end{aligned}
$$

\subsection{IMPES Type Algorithm}

The given nonlinear system of equations can be linearized by solving sequentially the pressure and saturation equations [11]. Application of this method for problem (3.1)-(3.4) gives the following algorithm:

\section{Implicit Pressure Equation}

$$
\left\{\begin{array}{l}
-\delta_{-} \delta_{+} P^{n, r}=\delta_{-} \delta_{+} \Sigma_{w}\left(\Phi^{n, r-1}\right) \\
P_{0}^{n, r}=P_{0}, \quad P_{M}^{n, r}=P_{a t m}
\end{array}\right.
$$

2. Mass Balance Equation

The difference scheme which uses the Enquist-Osher numerical flux $[5 ; 12]$ takes the following conservative form:

$$
\left\{\begin{array}{l}
\frac{\Phi_{j}^{n, r}-\Phi_{j}^{n}}{\tau_{n}}+\frac{F\left(v_{j+\frac{1}{2}}^{n, r}, \Phi_{j+1}^{n, r}, \Phi_{j}^{n, r}\right)-F\left(v_{j-\frac{1}{2}}^{n, r}, \Phi_{j}^{n, r}, \Phi_{j-1}^{n, r}\right)}{h}=0 \\
\Phi_{0}^{n, r}=\Phi_{r}, \quad \Phi_{M}^{n, r}=\Phi_{m}
\end{array}\right.
$$

where the numerical flux $F$ is defined as above. 


\subsection{Numerical Experiments}

The simulations presented in this section use the values of parameters given in [7], where the infiltration of a thermosetting resin in a network of glass fibers is considered.

The dependence of the permeability on the volume ratio is assumed to be given by

$$
\left.K\left(\Phi_{s}^{w}\right)=K_{0} e^{-16\left(\Phi_{s}^{w}-\Phi_{r}\right.}\right) .
$$

The stress-strain relations are given by

$$
\begin{aligned}
& \Sigma_{w}\left(\Phi_{s}^{w}\right)=\Sigma_{0}^{w}\left(e^{26.4 \Phi_{s}^{w}}-e^{26.4 \Phi_{r}}\right), \\
& \Sigma_{d}\left(\Phi_{s}^{d}\right)=\Sigma_{0}^{d}\left(e^{25 \Phi_{s}^{d}}-e^{25 \Phi_{r}}\right) .
\end{aligned}
$$

The resin viscosity and the function describing the curing process have been taken of the following type:

$$
\begin{aligned}
& \mu(\delta, \theta)= \begin{cases}\mu_{0} e^{18000 / R \theta}\left(\frac{0.1}{0.1-\delta}\right)^{1.5+\delta} & \text { if } \delta<0.1, \\
\infty & \text { otherwise, }\end{cases} \\
& \mu_{0}=2.78 \cdot 10^{-4}(\mathrm{~J} / \mathrm{mol}), \\
& f_{c}(\delta, \theta)=\left(c_{1} \exp \left(-\frac{E_{1}}{R \theta}\right)+c_{2} \exp \left(-\frac{E_{2}}{R \theta}\right) \delta^{0.3}\right)(1-\delta)^{1.7}, \\
& c_{1}=378330, c_{1}=678330, E_{1}=54418, E_{2}=50232,
\end{aligned}
$$

where $R$ is the $g$.

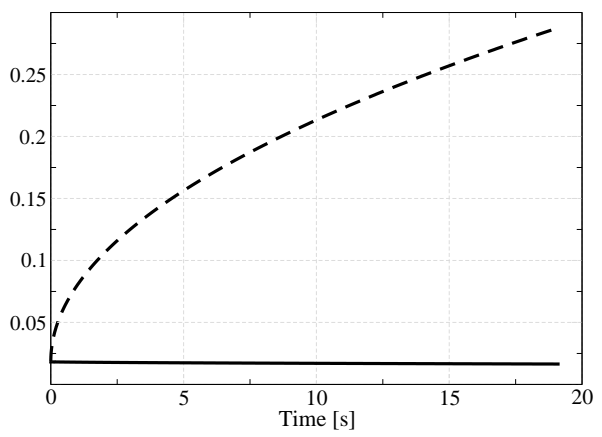

Figure 2. Positions of free boundaries: $x_{e}(t)$ (solid line) and $x_{i}(t)$ (dashed line).

In order to test the proposed finite-difference scheme we simulated the infiltration process which is driven by a constant pressure $0.1 M P a$. After the 
application of the pressure, the preform initially is compressed from $300 \mathrm{~mm}$ to $282 \mathrm{~mm}$. Figure 2 shows the evolution in time of $x_{e}(t)$ and $x_{i}(t)$.

As it is expected from the one-phase model, the interface $x_{i}(t)$ moves as $\sqrt{t}$, at least for the initial time interval.

Figure 3 gives the evolution of the solid volume fraction at different time moments.

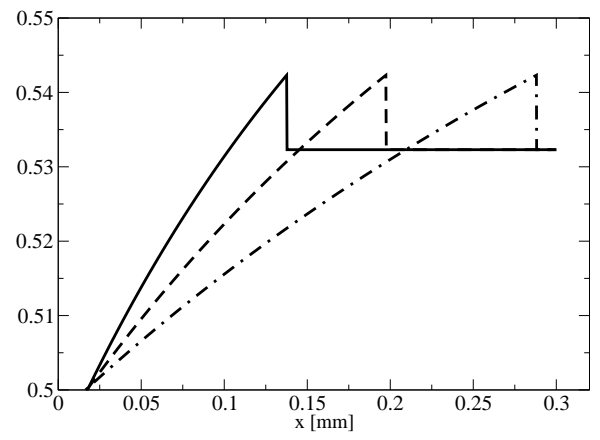

Figure 3. Solid volume fraction $\Phi_{s}(x, t): t=3.73$ (solid line), $t=8.43$ (dashed line), $t=19.16$ (dotted line).

\section{ACKNOLEDGEMENTS}

The authors wish to thank to Dr. V.Starikovičius for some valuable suggestions and discussions of these results.

\section{REFERENCES}

[1] D. Ambrosi and L. Preziosi. Modelling injection moulding processes with deformable porous preforms. SIAM J. Appl. Math., 61, $22-42,2000$.

[2] M. Bruschke, S. Advani and R. Parnas. Resin Transfer Moulding in Polymeric Composites, Ch.12. In: Flow and Rheology in Polymer Composites Manufacturing, 465 - 515, 1994.

[3] R.H. Christopher and S. Middleman. Power law flow through a packed tube. Ind. Eng. Chem. Fund., 4, 422 - 430, 1965.

[4] W.K. Chui, J. Glimm, F.M. Tangerman, A.P. Jardine, J.S. Madsen, T.M. Donnellan and R. Leek. Process modeling in resin transfer molding as a method to enhance product quality. SIAM Rev., 39, $714-727,1997$.

[5] B. Enquist and S. Osher. One-sided difference approximations for nonlinear conservation laws. Math. Comp., 36, $321-351,1981$.

[6] A. Farina. Filtration problems in various industrial processes. In: Filtration in porous media and industrial application. Springer, $79-126,2000$.

[7] A. Farina and L. Preziosi. Deformable porous media and composite manufacturing. In: Heterogeneous solids: micromechanics, modelling methods and simulations, Birkhauser, Boston, 2000.

[8] A. Farina and L. Preziosi. Infiltration process in composite materials manufacturing: modeling and qualitative results. In: Complex flows in industrial processes. Birkhauser, $281-306,2000$. 
[9] A. Farina and L. Preziosi. Non-isothermal injection moulding with with resin cure and preform deformability. Composites A., 31, 1355 - 1372, 2000.

[10] R. Greenkorn. Flow phenomena in porous media. Fundamentals and applications in petroleum, water and food production. Inc., NY and Basel, 1983.

[11] R. Helmig. Multiphase Flow and Transport Processes in the Subsurface. Springer, Berlin, Heidelberg, 1997.

[12] K.H. Karlsen, N.H. Risebro and J.D. Towers. Upwind difference approximations for degenerate parabolic convection-diffusion equations with a discontinuous coefficient. IMA J. Num. Anal., 22, 623 - 664, 2002.

[13] I.S. Liu. On chemical potential and incompressible porous media. J. Mech., 19, 327 $342,1980$.

[14] An. Mikelic. Homogenization theory and applications to filtration through porous media. In: Filtration in porous media and industrial application. Springer, $127-214$, 2000.

[15] D. Munaf, S. Wineman, K.R. Rajagopal and D.W. Lee. A boundary value problem in ground water motion analysis - comparison of the prediction based on Darcy's law and the continuum theory of mixture. Mat. Models Methods Appl. Sci., 3, 231 - 248, 1993.

[16] R.S. Parnas and F.R. Phelan. The effects of heterogenous porous media on mold filing in resin transfer molding. SAMPLE Quarterly, 22, 53 - 60, 1991.

[17] L. Preciozi. The theory of deformable porous media and its application to composite material manufacturing. Survey of Mathematics for Industry, 6, 167 - 214, 1996.

[18] T. Sadiq, S. Advany and R. Parnas. Experimental investigation of transverse flow through aligned cylinders. Int. J. Multiphase Flow, 21, 755 - 774, 1995.

[19] M.K. Um and L.J. Lee. A study of the mold filling in resin transfer molding. Polymer Science and Engineering, 31, 765 - 771, 1991.

\section{Polimerų filtracijos uždavinio skaitinis sprendimo algoritmas}

\section{R. Čiegis, O. Iliev}

Šiame darbe nagrinèjamas skystu polimeru filtracijos uždavinio skaitinis sprendimo algoritmas. Matematinis modelis yra aprašomas netiesinių diferencialinių lygčių dalinėmis išvestinemmis sistema. Dèl deformacijų filtracijos metu keičiasi uždavinio apibrèžimo srities geometrija, be to skysčio filtracijos frontas irgi juda, todèl formuluojamos dvi Stefano tipo kraštinès sąlygos. Diskrečioji aproksimacija gaunama baigtinių tūrių metodu.

Taip pat darbe sprendžiamas atvirkštinis mežiagos laidumo koeficiento nustatymo uždavinys. Gautos išreikštinès formulès Niutoninio ir apibendrinto Niutoninio skysčių tekejjimams. Šie teoriniai rezultatai palyginti su eksperimentiniais matavimų rezultatais. 